\title{
Nonsurgical integrative inpatient treatments for symptomatic lumbar spinal stenosis: a multi-arm randomized controlled pilot trial
}

This article was published in the following Dove Medical Press journal: Journal of Pain Research

\begin{abstract}
Kiok Kim, ${ }^{1, *}$ Kyung-Min Shin, ${ }^{2, *}$ Christy L Hunt, ${ }^{3}$ Zhen Wang, ${ }^{4}$ Brent A Bauer, ${ }^{5}$ Ojin Kwon, ${ }^{2}$ Jun-Hwan Lee, ${ }^{2,6}$ BokNam Seo, ${ }^{7}$ So-Young Jung, ${ }^{2}$ Yousuk Youn,' Sang Ho Lee,' Jung Chul Choi,' Jae Eun Jung,, Jaehong Kim,' Wenchun $\mathrm{Qu},{ }^{3,9}$ Tae-Hun Kim, ${ }^{10}$ Jason S Eldrige 9
\end{abstract}

'Department of Spine Center, Mokhuri Neck \& Back Hospital, Seoul, South Korea; ${ }^{2}$ Clinical Medicine Division, Korea Institute of Oriental Medicine, Daejeon, South Korea; ${ }^{3}$ Department of Physical Medicine and Rehabilitation, Mayo Clinic, Rochester, MN, USA; ${ }^{4}$ Robert D. and Patricia E. Kern Center for the Science of Health Care Delivery, Mayo Clinic, Rochester, MN, USA; ${ }^{5}$ Department of Internal Medicine, Mayo Clinic, Rochester, MN, USA

${ }^{6}$ Korean Medicine Life Science, Campus of Korea Institute of Oriental Medicine, University of Science \& Technology (UST), Daejeon, South Korea; ${ }^{7}$ Future Medicine Division, Korea Institute of Oriental Medicine, Daejeon, South Korea; ${ }^{8}$ Hongik Neurosurgery Hospital, Seongnam, South Korea; 'Department of Anesthesiology, Division of Pain Medicine, Mayo Clinic, Rochester, MN, USA; ${ }^{10}$ Korean Medicine Clinical Trial Center, Korean Medicine Hospital, Kyung Hee University, Seoul, South Korea

*These authors contributed equally to this work

Correspondence: Jason S Eldrige

Department of Anesthesiology, Division of Pain Medicine, Mayo Clinic, 200 First Street Southwest, Rochester, MN 55905, USA

Tel +I 5072849699

$\mathrm{Fax}+\mathrm{I} 5072667732$

Email Eldrige.jason@mayo.edu
Background: Lumbar spinal stenosis (LSS) is a chronic condition that causes low back pain and neurogenic claudication, often resulting in significant limitation of daily activities. In this open-label randomized controlled pilot study, we assessed the safety and feasibility of 4-week novel integrative inpatient treatments for LSS.

Methods: Thirty-six symptomatic LSS patients were randomly and equally allocated to one of the three groups: Mokhuri Chuna treatment 1 (MT1) group, Mokhuri Chuna treatment 2 (MT2) group, or conventional management treatment (CMT) group. MT1 patients were treated with herbal medication, Mokhuri Chuna, and acupuncture, and received daily physician consultation; MT2 patients were treated with Mokhuri Chuna and acupuncture without any herbal medication, and received daily physician consultation; and CMT patients received conventional pain management therapy that included epidural steroid injection, oral NSAID, and muscle relaxant medication, along with daily physiotherapy. The primary outcome of this pilot study was safety as measured by the type and incidence of adverse events (AEs). The secondary outcome measures included VAS score for low back pain and leg pain, Oswestry Disability Index, Oxford Claudication Score (OCS), walking capacity on a $50 \mathrm{~m}$ flat track and treadmill, and EuroQol-5D score. Magnetic resonance imaging was also performed up to 6 months after treatment cessation. Results: Thirty-four treated patients were included in the analysis, based on the modified intention-to-treat principle. No serious AEs were observed or reported. Compared to the CMT group, the MT1 and MT2 groups did show significant improvement at 3 and 6 months in various domains, including pain (VAS score for leg and back pain) and function (OCS and treadmill walking).

Conclusion: These novel multimodal integrative treatments for LSS are both clinically safe and logistically feasible. Larger, adequately powered randomized controlled trials will be necessary to assess comparative efficacy and thoroughly analyze the cost-effectiveness of each treatment approach.

Clinical trial registration number (CRIS): KCT0001218.

Keywords: lumbar spinal stenosis, complementary and alternative medicine, acupuncture, Chuna, epidural steroid injection, low back pain

\section{Background}

Lumbar spinal stenosis (LSS) can cause neurogenic claudication with discomfort of the buttock, thigh, and lower leg, which is classically exacerbated by lumbar extension and improved by spinal flexion. ${ }^{1,2}$ The leading cause of LSS is degenerative changes in various structures around the spinal canal or neural foramina including the vertebral discs, facet joints, and ligamentum flavum. ${ }^{1,2}$ 
LSS and its inducement of neurogenic claudication are the most common reasons for spinal surgery among individuals aged 65 years or older in the US. ${ }^{3}$ More than 37,000 laminectomies were performed in the US in 2007 for Medicare patients, resulting in total hospital costs approximating $\$ 1.65$ billion. ${ }^{2}$ LSS has high prevalence internationally as well, with the National Health Insurance Statistical Yearbook of South Korea designating LSS (ICD code: M48) as the eighth most frequent cause of admission among all inpatients during 2015. ${ }^{4}$ The South Korean medical costs due to LSS also increased from KRW 412.4 billion in 2011 to KRW 593.6 billion in $2015 .{ }^{4,5}$

LSS can be managed either surgically or nonsurgically. Though the overall spine surgery rate decreased slightly from 2002 to 2007 in the US, fusion surgeries increased 15 times over that same timeframe. ${ }^{3}$ A 2011 systematic review of randomized controlled trials (RCTs) comparing surgical to nonsurgical treatment for LSS indicates that surgical treatment had more positive results than nonsurgical interventions in regard to pain, disability, and quality of life at 3-6 months postoperatively, with effects persisting for 2-4 years. ${ }^{6}$ However, more recent data from the current 2016 Cochrane systematic review provide no clear conclusion about the benefits of surgical vs nonsurgical treatments for LSS. This study identified seven randomized and quasi-randomized controlled studies comparing surgical treatment for LSS to nonsurgical treatment appropriate for inclusion and analysis. This Cochrane analysis also reports a high rate of surgical complications ranging from $10 \%$ to $24 \%$ including such risks as spinous process fracture, reoperation, and death due to pulmonary edema. ${ }^{7}$ Furthermore, any need for reoperation exposes patients to additional potential consequence, including the development of acute postoperative problems, continued progression of degenerative changes, and persistent pain after surgery. ${ }^{8}$ A retrospective cohort analysis of Medicare claims in the US reported a reoperation rate of $11 \%$ at 4 years, ${ }^{8}$ while a retrospective cohort study using a national health insurance database in South Korea reported a reoperation rate of $4.7 \%$ within 3 months and $12.5 \%$ within 4 years. ${ }^{9}$ The attendant medical risk and additional financial costs associated with reoperation pose significant challenges for both patient and payer alike.

The natural history of this disease may at times be indolent or quiescent, with a prior 49-month observational study of untreated LSS revealing no symptomatic change in 70\% of the LSS cases; spontaneous improvement was actually demonstrated in $15 \%$ of these patients, with worsening/ progressive disease exhibited in the balance. ${ }^{10}$ Consequently, nonsurgical treatments are often recommended as a first-line therapy for LSS, with surgical treatments being reserved for patients who do not improve with conservative care. ${ }^{1,2}$ Immediate surgery should be recommended only when severe neurologic symptoms, such as myelopathy, develop or if the patient's pain is truly intractable and unbearable. ${ }^{10}$

Nonsurgical pain management therapy may include exercise, manual therapy, physical therapy, steroid epidural injections, and pharmacologic interventions. In South Korea, herbal medicine, acupuncture, moxibustion, and manipulation are often used for various pain conditions, including low back pain, with some reported success. ${ }^{11-14}$ However, definitive conclusions cannot be drawn about the effects of those treatments due to low-quality evidence and the small number of studies published. ${ }^{15,16}$ Studies examining the effectiveness of acupuncture suggest that it may be effective in the relief of low back pain, ${ }^{17,18}$ is well-tolerated in terms of side effects, and is cost effective. ${ }^{19}$

Traditional Chuna (Tuina in Mandarin) is a kind of spinal manipulative technique which consists of strong joint thrusts with mobilization of the spine and pelvis which is considered inappropriate for treatment of symptomatic LSS in elderly patients. ${ }^{20}$ Mokhuri Chuna is a particular style of Chuna that is gentler than traditional Chuna and safe for elderly patients with symptomatic LSS, and is the primary nonsurgical treatment for LSS examined in this clinical trial. It is designed to restore the muscle balance of the paraspinal musculature, relaxing muscular tension and restoring the function of the whole spine via subtle and soft manipulative movement. ${ }^{14}$ It involves manual manipulation of soft tissue utilizing a specially designed table such that patients may be positioned to allow for slight flexion of the thoracolumbar region. A retrospective study of 33 patients with LSS reported improvement of pain and walking duration at 1 year after 1 month of inpatient treatment using Mokhuri Chuna, acupuncture, and herbal medication with no reported adverse events (AEs), ${ }^{21}$ suggesting this treatment may be safe and effective. This is the first prospective clinical trial to date investigating the use of this therapy in patients with LSS.

In addition to Mokhuri Chuna, participants in one arm of the clinical trial received herbal medication widely used in traditional South Korean medicine for hundreds of years for the treatment of musculoskeletal conditions. These products are well known in East Asian herbal medicine. All herbs were used in equal quantities, including Geranium thunbergii, Saposhnikovia Radix, Acanthopanacis Cortex, Achyranthis Radix, Eucommiae Cortex, and Rhizoma Cibotii. Rhizoma Cibotii is known to exhibit antioxidant properties in vitro, and its clinical applications include relief of low back and leg 
pain..$^{22}$ Eucommiae Cortex exerts anti-inflammatory effects via downregulation of TLR4 pathways, which may be responsible for its therapeutic benefits in inflammatory musculoskeletal conditions. ${ }^{23}$ Acanthopanacis Cortex has been shown to increase bone mass in preclinical studies via downregulation of $\mathrm{RANKL}^{24}$ and to also exhibit anti-inflammatory properties including decreased nitrous oxide and prostaglandin E2 production. ${ }^{25}$ G. thunbergii exerts anti-inflammatory effects via inhibition of lipopolysaccharide- and interferon- $\gamma$-induced expression of pro-inflammatory genes. ${ }^{26}$

This pilot study examines the feasibility, safety, and potential efficacy of nonsurgical South Korean integrative interventions for LSS. Our study also provides the clinical foundation for consideration of larger-scale RCTs needed to more definitively answer the questions of comparative efficacy, cost-effectiveness, and risks and benefits and to clarify the therapeutic algorithm for best patient care.

\section{Methods}

\section{Study design}

This study was a single-center, randomized, controlled, conventional treatment pilot study investigating the safety and feasibility of a 4-week South Korean integrative inpatient treatment program for patients with severe symptomatic LSS. The aim of this study was to assess the feasibility of the nonsurgical integrative Mokhuri Chuna treatments and to analyze the preliminary clinical effectiveness and safety comparing two different types of Mokhuri Chuna treatments and conventional management treatment (CMT) within inpatient care units. The trial was carried out with three groups:

MT1 group: Mokhuri Chuna, acupuncture, and herbal medication

MT2 group: Mokhuri Chuna and acupuncture only

CMT group: conventional pain management treatment including oral medications (NSAID + muscle relaxant), epidural steroid injections, and physiotherapy

The study was conducted in accordance with the Declaration of Helsinki at Mokhuri Oriental Medical Hospital, Seoul, South Korea, from April 2015 to November 2016. Protocol approval was established prior to the recruitment of patients by the Institutional Review Board of Mokhuri Hospital (MHNBH-14001). A detailed version of the study methodology has been published elsewhere. ${ }^{27}$

\section{Participants}

Patients aged 19-77 years old with at least 1 year of low back pain, radicular leg pain, or leg discomfort consistent with LSS diagnosed through computed tomography (CT) or magnetic resonance imaging (MRI) were recruited through print and online advertisements. Patients who had been recommended for surgical treatments including decompression and/or fusion surgery following treatment failure as defined by inadequate response to at least 3 months of nonsurgical treatment for LSS were included in the study. Included patients endorsed neurogenic claudication symptoms during a 5-minute treadmill walking test with a speed of at least $1.5 \mathrm{miles} /$ hour. Individuals with spinal disease including ankylosing spondylitis or spinal osteomyelitis, metabolic bone disease including severe osteoporosis, peripheral joint disease, or vascular claudication, and those who were undergoing treatments that could affect the effect of interventions were excluded.

All patients were recruited between April 2015 and April 2016. After giving written informed consent, patients were allocated randomly based on a computer-generated sequence to one of the treatment groups in a 1:1:1 ratio, stratified by severity of spondylolisthesis. An independent statistician prepared opaque sealed envelopes and assigned patients to ensure appropriate and unbiased allocation.

\section{Intervention}

All patients in the MT1 and MT2 groups were treated with Mokhuri Chuna once daily, for 5 days per week, according to the protocol described in the previous section. For the purposes of the current study, these treatments were standardized for all subjects. During Mokhuri Chuna treatment, patients are placed prone on an automated table which has the ability to mechanically flex and distract the lumbar spine (Ergo Style ${ }^{\text {TM }}$ FX-5820 Table; Chattanooga Group, affiliate of DJO International, Guildford, Surrey, England (Figure 1). The table is adjusted to flex to a comfortable position, no more than $15^{\circ}$, and its speed may be adjusted according to the patient's breathing pattern, generally 8-15 times per minute. While the table is set for autoflexion, the practitioner engages in concomitant soft tissue manipulation which includes distraction and stretching of the back and gluteal muscles including the latissimus dorsi, rhomboid, quadratus lumborum, gluteus medius and minimus, and paraspinal musculature. Manual manipulation is performed in both the lumbar and thoracic spinal regions, generally for 5-6 minutes per session. Patients are then placed into the lateral recumbent position, and a gentle lumbar roll manipulative technique is engaged first ipsilaterally and then on the contralateral side. The terminal maneuvers position the patients supine, where each hip joint then undergoes direct flexion, abduction, and external rotation three to four times on each side (Figures 2-4). 


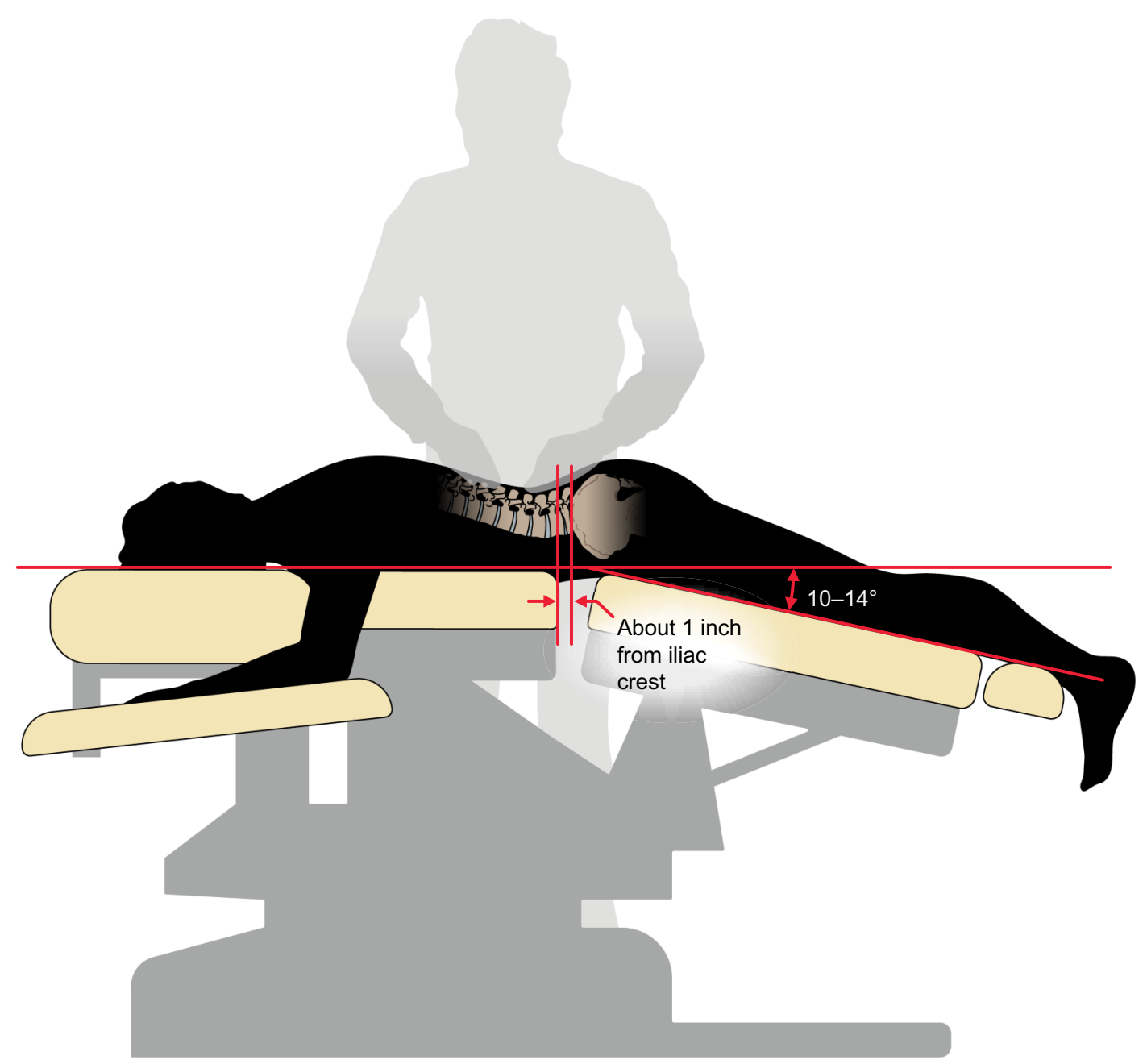

Figure I Mokhuri Chuna treatment illustration.

Notes: During Mokhuri Chuna treatment, patients are placed prone on an automated table which has the ability to mechanically flex and distract the lumbar spine. While the table is set for autoflexion, the practitioner engages in concomitant soft tissue manipulation which includes distraction and stretching of the back and gluteal muscles. Manual manipulation is performed in both the lumbar and thoracic spinal regions, generally for 5-6 minutes per session.

In the MT1 group, $110 \mathrm{~g}$ of Gang-Chuk Tang was administered three times a day. Gang-Chuk Tang is an herbal concoction consisting of Eucommiae Cortex, Achyranthis Radix, Rhizoma Cibotii, Sorbus commixta, G. thunbergii, Saposhnikovia Radix, and Acanthopanacis Cortex in equal portions. In addition, Mokhuri Chuna therapy was provided daily, which consisted of relaxation and mobilization of lumbar joint and back muscle, using Ergo Style ${ }^{\mathrm{TM}}$ FX-5820 Table (Chattanooga Group) ${ }^{28}$ The MT1 group also underwent daily acupuncture treatment on LI4, ST36, LV3, BL22, BL23, BL24, BL25, and Ashi points $(0.25 \times 40 \mathrm{~mm}$ disposable stainless steel needles; Dongbang Acupuncture Co., Choongchungnamdo, South Korea). Lastly, MT1 patients received consultation on precautions related to daily activ- ity and stepwise walking training for the entire 4 weeks of therapy. The treatment was administered by a certified Korean medical doctor who has over 10 years of clinical experience.

In the MT2 group, Mokhuri Chuna, acupuncture, and physician consultation were offered in the same manner and dosage as the MT1 group with the exception that all herbal medications were withheld. In the CMT group, oral analgesic therapy (aceclofenac $100 \mathrm{mg}$ twice daily and eperisione hydrochloride $50 \mathrm{mg}$ three times daily for 28 days) and three interlaminar epidural steroid injections (5 $\mathrm{mg}$ of dexamethasone per injection) at the level of the affected spinal region over a 4-week period were administered. ${ }^{29}$ All patients in the CMT group also received physiotherapy including use of a heating pad (Stemkorea Co., Seoul, South Korea) and 


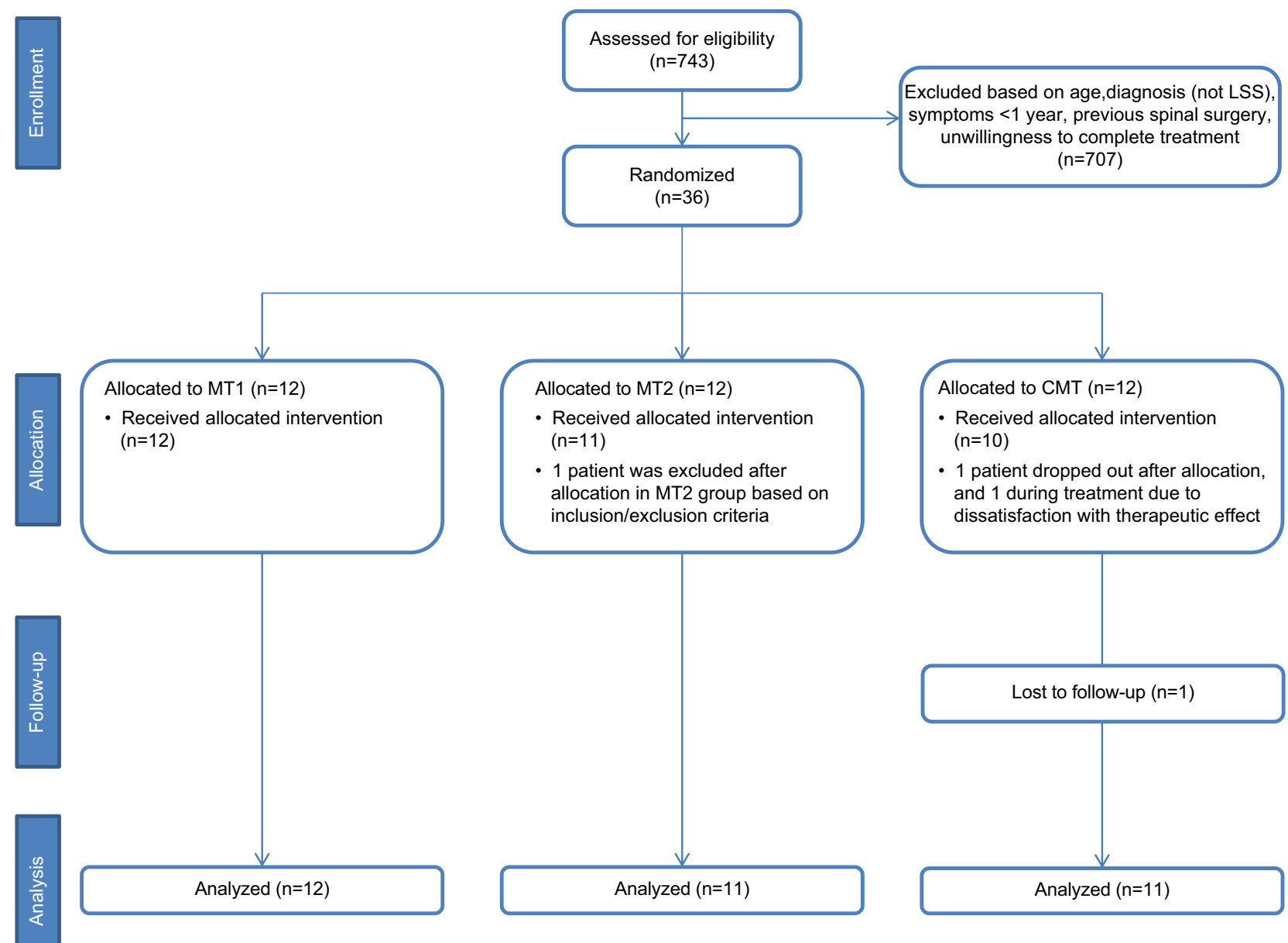

Figure 2 Consort enrollment diagram.

Notes: A total of 743 potential subjects were screened during the study period. Of these, 36 patients fulfilled the inclusion and exclusion criteria, consented to the study, and were randomized to one of the three treatment groups. Two patients (one in the CMT group and one in the MT2 group) withdrew before the start of the treatments and were not included in the analyses. One additional patient in the CMT group dropped out during the treatment phase, and another patient in the CMT group was lost to follow-up. Thirty-four patients were included in the statistical analysis (I 2 in the MTI group, II in the MT2 group, and II in the CMT group).

Abbreviations: CMT, conventional management treatment; LSS, lumbar spinal stenosis; MTI, Mokhuri Chuna treatment I; MT2, Mokhuri Chuna treatment 2.

\section{VAS, low back pain (mean scores)}

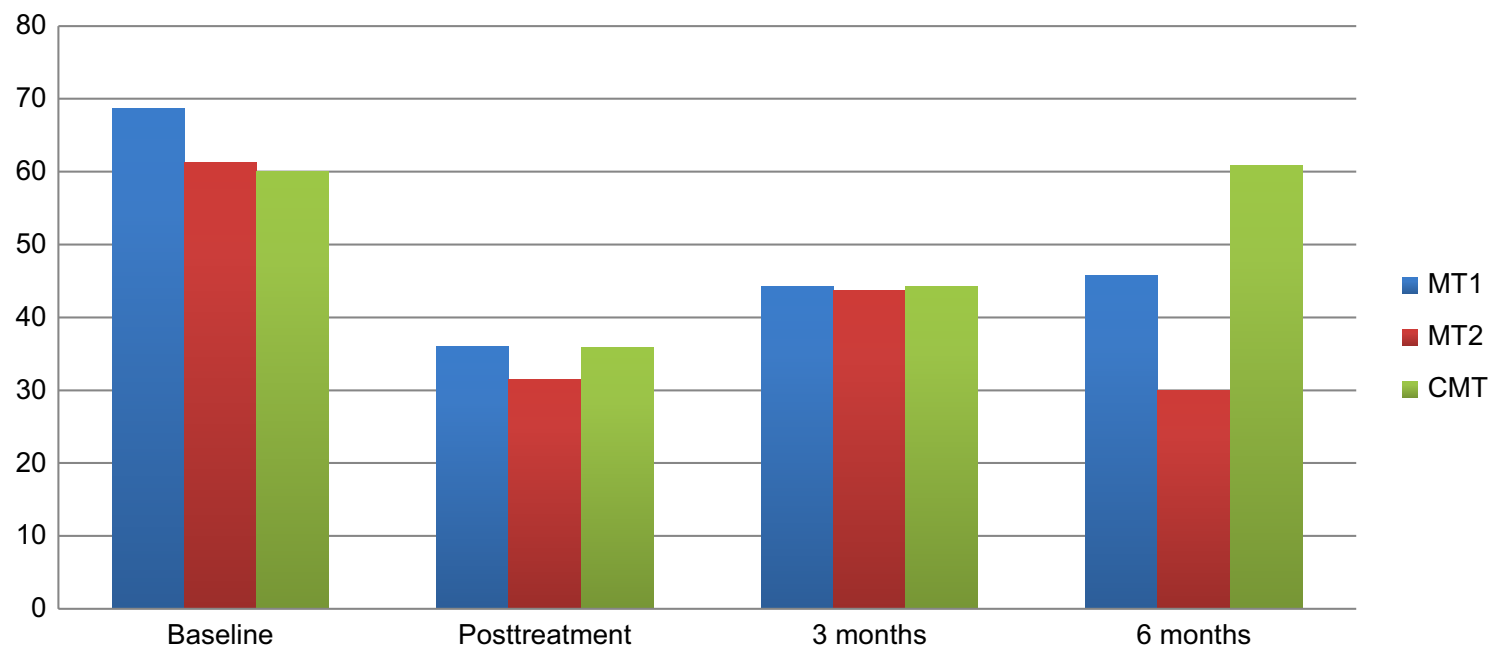

Figure 3 Mean VAS scores for low back pain at baseline, immediately posttreatment, and at 3- and 6-month follow-up visits for each cohort. Abbreviations: CMT, conventional management treatment; MTI, Mokhuri Chuna treatment I; MT2, Mokhuri Chuna treatment 2. 
VAS, leg pain (mean scores)

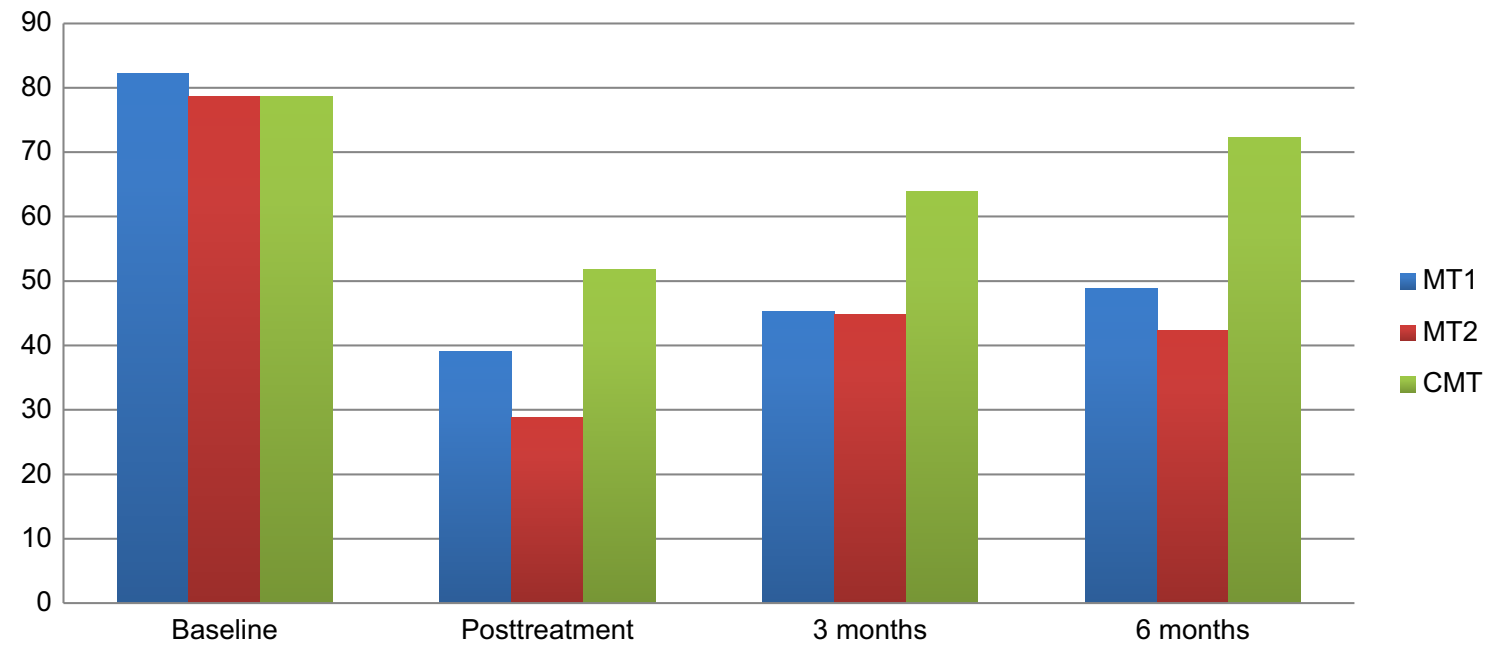

Figure 4 Mean VAS scores for leg pain at baseline, immediately posttreatment, and at 3-and 6-month follow-up visits for each cohort. Abbreviations: CMT, conventional management treatment; MTI, Mokhuri Chuna treatment I; MT2, Mokhuri Chuna treatment 2.

transcutaneous electrical nerve stimulator (TENS; Homer Ion Laboratory Co. Ltd., Tokyo, Japan) and deep tissue heating therapy (Radio Derm; RoboMax Co, Seongnam, South Korea) applied at the affected region five times per week for 4 weeks. One patient in the CMT group did not receive any epidural steroid injections due to failure to respond in the past. Detailed information about these interventions was reported in the previously published protocol of this study. ${ }^{27}$

\section{Safety and monitoring}

AEs were assessed daily during the treatment period and at each follow-up visit. The patients were instructed to contact study staff immediately should they experience any side effects or AEs in between follow-up visits. Blood analysis, urine analysis, and electrocardiography were performed in all subjects at baseline prior to treatment, and also at the end of 4 weeks of therapy.

\section{Outcome measures}

The primary purpose of this study was to assess the safety and feasibility of Mokhuri Chuna, a nonsurgical technique, for treatment of symptomatic LSS in an inpatient setting. The secondary outcome measures included assessment of pain and function of LSS patients; this was evaluated before and after the 4-week participation period and also at 3- and 6-month follow-up intervals. The VAS score for average low back and leg pain during 1 week was used to evaluate pain. The Oswestry Disability Index (ODI) ${ }^{30}$ was used to assess function and disability related to low back pain, while the Oxford Claudication Score (OCS) was used to assess formal neurogenic claudication symptoms. ${ }^{31}$ For the assessment of physical function, a treadmill walking test was utilized, during which the time taken for pain development in the lower limbs during walking on a flat treadmill at a speed of $1.5 \mathrm{miles} / \mathrm{hour}$ and on a $50 \mathrm{~m}$ flat track at a subject-determined comfortable maximum speed was recorded. The EuroQol-5D (EQ-5D) was used to assess the quality of life. ${ }^{32}$ In this study, we used the validated Korean-language version of each assessment tool except OCS which was not available at the time of the study. Radiologic testing, including lumbar spine MRI and lumbar $\mathrm{X}$-ray imaging, was performed at the screening visit and 3 and 6 months after treatment in order to assess any dimensional change in the central spinal canal and/or to elucidate any sign of structural instability. This testing allowed formal quantification of the anterior-to-posterior diameter of the spinal canal, which was measured at baseline and after the 3- and 6-month treatment periods. Independent outcome assessors who did not participate in the treatments conducted all evaluations of pain and function in the participants. Furthermore, an independent radiologist, who was blinded to group allocation, provided all radiologic interpretation.

\section{Statistical analysis}

No formal power calculation was conducted for this pilot study, given that the primary goals were to assess the safety and feasibility of the interventions. Statistical analysis was performed by using the modified intention-to-treat principle after imputation of the missing values by the last-observationcarried-forward method. Patients who withdrew before the start of the treatments were not included in the analyses. Baseline 
characteristics of the participants were described as mean (SD) for continuous variables and as frequency (percentage) for categorical variables. We compared difference between groups using Kruskal-Wallis test for continuous variables, and chi-squared test and Fisher's exact test for categorical variables. All statistical analyses were performed using Stata version 15.1 (StataCorp LLC, College Station, TX, USA) at a significance level of $5 \%$ using a two-tailed test methodology.

\section{Results}

A total of 743 potential subjects were screened during the study period. Of these, 36 patients fulfilled the inclusion and exclusion criteria, consented to the study, and were randomized to one of the three treatment groups (Figure 2). Two patients (one in the CMT group and one in the MT2 group) withdrew before the start of the treatments and were not included in the analyses. One additional patient in the CMT group dropped out during the treatment phase, and another patient in the CMT group was lost to follow-up. Thirty-four patients were included in the statistical analysis (12 in the MT1 group, 11 in the MT2 group, and 11 in the CMT group). The average age of the patients was 64 years (SD: 5.3) and $66.7 \%$ were women, with an average participant body mass index (BMI) of 24 (SD: 2.9). There were no statistically significant differences noted among groups in the following baseline demographics: sex, age, BMI, exercise, tobacco use, alcohol use, or employment (Table 1).

\section{Primary outcome}

\section{Adverse events}

Twenty-four cases of AEs were reported during 1,054 treatment sessions, and there were no statistically significant differences noted in the incidence of AEs between the three groups (MT1: eight cases [2.15\%], MT2: nine cases [2.64\%], CMT: seven cases [2.05\%]; $P=0.86)$. AEs included respiratory symptoms such as rhinitis $(\mathrm{n}=9)$, coughing $(\mathrm{n}=2)$, and pneumonia $(\mathrm{n}=1)$, pain including headache $(\mathrm{n}=1)$, low back pain $(n=1)$, and ankle sprain $(n=1)$, and other general pain $(n=9)$. All reported AEs were determined to be unrelated to treatment. All AEs improved spontaneously during the participation period (Table 2). There were no serious AEs related to treatment during the study period. Overall, patients tolerated the Mokhuri Chuna treatments, acupuncture, and herbal medication well, and no patients terminated treatment due to intolerance of side effects.

\section{Secondary outcomes}

Table 3 summarizes the intergroup differences among the experimental treatment groups (MT1, MT2) and CMT group assessed at baseline, posttreatment, and at 3- and 6-month follow-up visits. At the immediate posttreatment visit, the only statistically significant intergroup difference was found in the VAS for leg pain between the MT2 (mean \pm SD: 28.82 \pm 27.46 ) and CMT (51.82 \pm 25.34$)$ groups $(P=0.04)$. At 3 months following treatment, there was no intergroup difference in the VAS for low back and leg pain. At 6 months after treatment, there were significant intergroup differences in the VAS for low back pain between the MT2 (30.00 \pm 13.48$)$ and CMT (60.82 \pm 18.62$)$ groups $(P=0.001)$, and in the VAS for leg pain between the MT1 (48.91 \pm 23.08$)$ and CMT $(72.27 \pm 16.72)$ groups $(P=0.01)$ and between the MT2 (42.36 \pm 21.29$)$ and CMT groups $(P=0.003)$. In terms of disability, no statistically significant intergroup differences were found among the three treatment arms at any time point

Table I Baseline demographic data

\begin{tabular}{|c|c|c|c|c|}
\hline Characteristics & MTI $(N=\mid 2)$ & MT2 $(N=I I)$ & CMT (N=II) & All cohorts \\
\hline Age, mean (SD), years & $65(4.8)$ & $66(3.6)$ & $62(6.5)$ & $64(5.3)$ \\
\hline Women, no. (\%) & $10(83.3)$ & $8(66.7)$ & $6(50.0)$ & $24(66.7)$ \\
\hline BMI, mean (SD) & $24(2.9)$ & $25(3.5)$ & $24(2.1)$ & $24(2.9)$ \\
\hline Smoker, no. (\%) & $0(0)$ & $0(0)$ & $\mathrm{I}(8.3)$ & $\mathrm{I}(2.8)$ \\
\hline Employed, no. (\%) & $4(33.3)$ & $3(25.0)$ & $3(25.0)$ & $10(27.8)$ \\
\hline LBP duration $\leq 12$ months, no. (\%) & $I(8.3)$ & $0(0)$ & $0(0)$ & $\mathrm{I}(2.8)$ \\
\hline LBP duration > 12 months, no. (\%) & II (9I.7) & $12(100)$ & $12(100)$ & $35(97.2)$ \\
\hline Leg pain duration $\leq 12$ months, no. (\%) & $\mathrm{I}(8.3)$ & $0(0)$ & $\mathrm{I}(8.3)$ & $2(5.6)$ \\
\hline Leg pain duration $>12$ months, no. (\%) & II (9I.7) & $12(100)$ & II (9I.7) & $34(94.4)$ \\
\hline SS diagnosis $\leq 12$ months, no. (\%) & $4(33.3)$ & $3(25.0)$ & $3(25.0)$ & $10(27.8)$ \\
\hline SS diagnosis $>12$ months, no. (\%) & $8(66.7)$ & $9(75.0)$ & $9(75.0)$ & $26(72.2)$ \\
\hline Treadmill tolerance time, mean (SD), seconds & $90(109.9)$ & $49(47.5)$ & $97(102.6)$ & $79(91.0)$ \\
\hline
\end{tabular}

Abbreviations: BMI, body mass index; CMT, conventional management treatment; LBP, low back pain; MTI, Mokhuri Chuna treatment I; MT2, Mokhuri Chuna treatment 2; SS, spinal stenosis. 
Table 2 Pooled data of adverse events

\begin{tabular}{|c|c|c|c|c|}
\hline Adverse events & MTI $(N=I 2)$ & MT2 $(\mathbf{N}=I I)$ & CMT (N=II) & Total \\
\hline Treatment-related adverse events & 0 & 0 & 0 & 0 \\
\hline \multicolumn{5}{|l|}{ Adverse events not related to treatment } \\
\hline Upper respiratory infection & 2 & 3 & 4 & 9 \\
\hline Lower respiratory infection & 0 & 2 & 1 & 3 \\
\hline Headache & 1 & 0 & 0 & 1 \\
\hline Low back pain (fracture due to fall) & I & 0 & 0 & I \\
\hline Ankle sprain & 0 & 1 & 0 & 1 \\
\hline Gingivitis & 2 & $\mathrm{I}$ & 0 & 3 \\
\hline Pruritus & 1 & 1 & 0 & 2 \\
\hline Dyspepsia & 1 & 0 & 0 & $\mathrm{I}$ \\
\hline Insomnia & 0 & 0 & 1 & I \\
\hline Constipation & 0 & 0 & 1 & I \\
\hline Onychomycosis & 0 & 1 & 0 & I \\
\hline
\end{tabular}

Abbreviations: CMT, conventional management treatment; MTI, Mokhuri Chuna treatment I; MT2, Mokhuri Chuna treatment 2.

Table 3 Significance associated with between-group differences in secondary outcome measures

\begin{tabular}{|c|c|c|c|c|}
\hline Visit & $P$-value, all groups & $P$-value, MTI vs MT2 & $P$-value, MTI vs CMT & $P$-value, $M T 2$ vs CMT \\
\hline \multicolumn{5}{|c|}{ VAS score, leg pain } \\
\hline Baseline & 0.63 & 0.69 & 0.27 & 0.82 \\
\hline Posttreatment & 0.72 & 0.54 & 0.76 & 0.45 \\
\hline 3 months & 0.98 & 0.90 & 0.85 & 0.95 \\
\hline 6 months & $0.01 * *$ & 0.36 & 0.14 & $0.001 * * *$ \\
\hline \multicolumn{5}{|c|}{ VAS score, low back pain } \\
\hline Baseline & 0.84 & 0.71 & 0.56 & 0.87 \\
\hline Posttreatment & 0.08 & 0.19 & 0.19 & $0.04 *$ \\
\hline 3 months & 0.19 & 0.95 & 0.12 & 0.11 \\
\hline 6 months & $0.01 * *$ & 0.58 & $0.01 * *$ & $0.003 * *$ \\
\hline \multicolumn{5}{|l|}{ ODI } \\
\hline Baseline & 0.65 & 0.52 & 0.95 & 0.34 \\
\hline Posttreatment & 0.87 & 0.54 & 0.90 & 0.77 \\
\hline 3 months & 0.57 & 0.29 & 0.62 & 0.58 \\
\hline 6 months & 0.09 & 0.60 & $0.05^{*}$ & 0.08 \\
\hline \multicolumn{5}{|l|}{ OCS } \\
\hline Baseline & 0.35 & 0.28 & 0.83 & 0.16 \\
\hline Posttreatment & 0.15 & 0.50 & $0.05^{*}$ & 0.25 \\
\hline 3 months & $0.05^{*}$ & 0.11 & $0.02 *$ & 0.28 \\
\hline 6 months & 0.14 & 0.60 & 0.08 & 0.11 \\
\hline \multicolumn{5}{|l|}{ Walking distance } \\
\hline Baseline & 0.36 & 0.20 & 0.76 & 0.25 \\
\hline Posttreatment & 0.72 & 0.44 & 0.54 & 0.97 \\
\hline 3 months & 0.06 & 0.11 & $0.03 *$ & $0.4 \mathrm{I}$ \\
\hline 6 months & $0.04 *$ & 0.20 & $0.01 * *$ & 0.22 \\
\hline \multicolumn{5}{|c|}{ Treadmill tolerance time } \\
\hline Baseline & 0.94 & 0.72 & 0.72 & 0.94 \\
\hline Posttreatment & 0.43 & 0.55 & 0.13 & 0.79 \\
\hline 3 months & 0.14 & 0.62 & $0.05 *$ & 0.17 \\
\hline 6 months & $0.04 *$ & 0.50 & $0.02 *$ & $0.04 *$ \\
\hline \multicolumn{5}{|l|}{ EQ-5D score } \\
\hline Baseline & 0.13 & 0.73 & 0.06 & 0.10 \\
\hline Posttreatment & 0.24 & 0.10 & 0.58 & 0.20 \\
\hline 3 months & 0.99 & 0.90 & 0.95 & 0.90 \\
\hline 6 months & 0.18 & 0.36 & 0.29 & 0.06 \\
\hline \multicolumn{5}{|l|}{ MRI findings } \\
\hline Screening & 0.66 & 0.54 & 0.39 & 0.55 \\
\hline Posttreatment & 0.55 & 0.58 & 0.36 & 0.39 \\
\hline 6 months & 0.25 & 0.67 & 0.18 & 0.14 \\
\hline
\end{tabular}

Notes: $* P \leq 0.05 . * * P \leq 0.01 . * * * P \leq 0.001$.

Abbreviations: CMT, conventional management treatment; EQ-5D, EuroQol-5D; MRI, magnetic resonance imaging; MTI, Mokhuri Chuna treatment I; MT2, Mokhuri Chuna treatment 2; OCS, Oxford Claudication Score; ODI, Oswestry Disability Index. 
based upon the measured ODI. The only statistically significant intergroup difference in terms of neurogenic claudication symptoms was found between the MT1 (18.75 \pm 6.52$)$ and CMT (25.82 \pm 6.24$)$ groups at 3 months as per Oxford Claudication Scoring $(P=0.02)$. Walking distance without pain was significantly different between the MT1 and CMT groups at both $3(P=0.03)$ and 6 months $(P=0.01)$ following treatment. Walking tolerance on treadmill was significantly different at 6 months between the MT1 and CMT groups $(P=0.02)$ and between the MT2 and CMT groups $(P=0.04)$. No intergroup differences were noted at any time point in terms of quality of life as assessed by the EQ-5D. In addition, no significant intergroup differences in terms of anterior-to-posterior diameter of the spinal canal measured on MRI were found.

\section{Discussion}

In this study, 4-week inpatient integrative treatments consisting of acupuncture, Mokhuri Chuna, and daily physician consultation with (MT1 group) or without herbal medication (MT2 group) were compared with the conventional pain management treatment (CMT group), which included epidural steroid injections, oral pharmacotherapy, and daily physiotherapy. Clinical outcomes for all three patient groups were tracked closely at four discrete intervals: baseline prior to therapy, and at 4 weeks, 3 months, and 6 months after treatment. Notably, we were able to demonstrate reassuring safety findings with few AEs (none serious). Interestingly, we were also able to show statistically significant improvement in leg pain as assessed by VAS at 6 months for the MT1 and MT2 groups (relative to the CMT group). Even more intriguing is the finding that both walking distance and treadmill tolerance continued to progressively improve in both the MT1 and MT2 groups at both 3- and 6-month follow-up visits. Conversely, the CMT group showed progressive decline in both of the aforementioned functional measures at the immediate posttreatment visit and at the 3- and 6-month assessments. While not definitively conclusive, this observation suggests that Mokhuri Chuna and/or acupuncture may confer long-lasting therapeutic benefits beyond the isolated treatment window, given that these were interventions unique to both MT1 and MT2 patient groups. The length of time over which patients in the MT1 and MT2 arms sustained trends toward improvement in pain and function is intriguing, suggesting that Mokhuri Chuna and/or acupuncture may improve the quality of life of patients with LSS treated nonoperatively.

Regarding our primary outcomes, recruitment was accomplished within 1 year of study initiation with a very small dropout rate during the treatment and follow-up periods. The aforementioned rapidity of recruitment and robust completion rate demonstrate high study feasibility in terms of ease of participation and follow-through for both patients and clinicians alike. In particular, there did not appear to be any patient adversity to 4 weeks of inpatient therapy; indeed, all dropouts (two) appeared to be due to dissatisfaction with treatment result and therapeutic expectation rather than intolerance to the duration of therapeutic course. By any objective measure, our AE rate of $2.28 \%$ is far less than the commonly reported $10 \%-24 \%$ complication rate associated with surgery. In addition, there were no serious AEs, and the vast majority of AEs were either non-painful or otherwise commonly found incidental symptoms (14 of 24) that did not appear to be causally linked to any of our treatments by any ostensible mechanism.

Our study's assessment of patient pain and function yielded a few interesting results, particularly longer-term 3and 6-month outcomes. After 4 weeks of treatment, the only significant difference noted was improved VAS score for leg pain in the MT2 group relative to the CMT group ( $P=0.04)$. VAS score was statistically improved for low back pain in the MT2 group (vs CMT) at 6 months, and also significantly lessened for leg pain in the MT1 and MT2 groups (vs CMT) at 6 months. From a functional standpoint, the most interesting observation was improved treadmill tolerance at 6 months in both the MT1 and MT2 groups (relative to CMT). Additionally, both walking distance and treadmill tolerance continued to improve at 3 months and again at 6 months in both MT1 and MT2 groups, while these metrics worsened in the CMT group at both time intervals. As mentioned previously, this raises the question of whether a therapy unique to the MT1 and MT2 groups (and not provided to the CMT group) conferred prolonged functional benefits to patients; the potential therapeutic candidates would seem to include either Chuna or acupuncture, though the study is not sufficiently designed to provide clear insight into this particular question. The design of the study precludes definitive analysis regarding the precise mechanism of these lasting effects beyond the treatment period. One hypothesis meriting further study is that these therapies may have facilitated improvement in musculature in treated patients, either directly or via pain reduction, leading to sustained functional improvements.

\section{Limitations}

First, this is a preliminary pilot study conducted to assess the safety and logistical feasibility of both the therapeutic interventions and the study design (primary objectives). Our study's sample size was not sufficiently large to draw definitive 
conclusions regarding the overall efficacy of therapy in the treatment groups; therefore, future studies should include larger number of patients sufficient to speak of therapeutic efficacy. Second, our use of multifaceted treatments within each group does provide convolution which makes it impossible to deduce whether or not specific treatments in isolation (such as herbal therapy alone) offer particularly enhanced efficacy relative to other treatments. Third, the patient demographics revealed a mean BMI of 24; this may make our study findings less relatable to practice environments in which morbid obesity is endemic. In clinical practice, a multimodal approach is a generally accepted standard of care for treatment of LSS patients, ${ }^{16}$ but a 4-week inpatientbased environment may not be common or available in many practice settings throughout the world. Though we did assess outcomes up to 6 months after treatment, degenerative LSS is a chronic condition which needs comparatively long-term care; 6 months is likely not enough time to fully evaluate the long-term effectiveness of interventions. From this perspective, we are now conducting a 2-year follow-up study with the participants of this study to assess the longer-term effects of our intensive integrative inpatient treatment program. Finally, we acknowledge that pain and response to treatment are inherently subjective, and cultural norms and expectations might impact treatment response. Another limitation of this study is that we did not collect data on expectation of treatment outcomes from study participants, which perhaps could have attenuated their response to treatment and reported outcomes.

\section{Conclusion}

This study illustrates that 4-week inpatient integrative treatment with acupuncture, Mokhuri Chuna, and daily physician consultation with or without herbal medication is clinically feasible with no reported serious AEs related to treatment. A trend of clinical improvement was observed for the treatment groups MT1 and MT2, with continued progressive improvement in walking distance and treadmill tolerance at both 3 and 6 months after treatment, compared to the CMT group. There was no significant difference in the frequency and type of AEs between groups, and no observed AEs were serious. Future full-scale RCTs would more precisely answer the remaining questions of comparative efficacy, cost-effectiveness, and risks and benefits to patients. Understanding the chronicity and debility of symptomatic LSS, the importance of interventions which improve functional capacity and thereby facilitate greater activity levels in patients is self-evident. We are very optimistic about the implication that some of our chosen therapies may endow patients with improved abilities for walking and treadmill tolerance over time. Moreover, given the great economic cost and significant morbidity associated with surgical interventions, it behooves all of us to continue the search for safe, effective, and fiscally responsible treatment alternatives for LSS patients.

\section{Acknowledgments}

This study was supported by the Korea Institute of Oriental Medicine (K15130), and by a grant of the Korea Health Technology R\&D Project through the Korea Health Industry Development Institute (KHIDI), funded by the Ministry of Health \& Welfare, South Korea (grant number: HI16C1625).

\section{Author contributions}

KK, KMS, JSE, BAB, JHL, BNS, SYJ, YY, SHL, JCC, JK, WQ, and THK conceived the study protocol and conducted the clinical study. OJK and ZW conducted the statistical analysis. CLH, JSE, BAB, THK, KK, KMS, JHL, JEJ, $\mathrm{JK}, \mathrm{WQ}$, and JY wrote and fully revised the manuscript in preparation for submission. All authors contributed to data analysis, drafting or revising the article, gave final approval of the version to be published, and agree to be accountable for all aspects of the work.

Three research institutions participated, including the spine center of Mokhuri Oriental Medical Hospital (one of the nationally certified spine specialized hospitals), the Korea Institute of Oriental Medicine, and the complementary and integrative medicine program of Mayo Clinic Foundation (Rochester, MN).

\section{Disclosure}

The authors report no conflicts of interest in this work.

\section{References}

1. Katz JN, Harris MB. Lumbar spinal stenosis. NEngl J Med. 2008;358(8): 818-825.

2. Lurie J, Tomkins-Lane C. Management of lumbar spinal stenosis. BMJ. 2016;352:h6234.

3. Deyo RA, Mirza SK, Martin BI, Kreuter W, Goodman DC, Jarvik JG, Trends JJG. Trends, major medical complications, and charges associated with surgery for lumbar spinal stenosis in older adults. JAMA. 2010;303(13):1259-1265.

4. Son MS, Seong SC. 2015 National Health Insurance Statistical Yearbook. Health Insurance Review \& Assessment Service, National Health Insurance Service; Wonju, South Korea. 2015.

5. Kim JD, Kang YK. 2011 National Health Insurance Statistical Yearbook. National Health Insurance Service, Health Insurance Review \& Assessment Service; Seoul, South Korea. 2011.

6. Kovacs FM, Urrútia G, Alarcón JD. Surgery versus conservative treatment for symptomatic lumbar spinal stenosis: a systematic review of randomized controlled trials. Spine. 2011;36(20):E1335-E1351.

7. Zaina F, Tomkins-Lane C, Carragee E, Negrini S. Surgical versus nonsurgical treatment for lumbar spinal stenosis. Spine. 2016;41(14):E857-E868. 
8. Deyo RA, Martin BI, Kreuter W, Jarvik JG, Angier H, Mirza SK. Revision surgery following operations for lumbar stenosis. J Bone Joint Surg Am. 2011;93(21):1979-1986.

9. Kim CH, Chung CK, Park CS, Choi B, Kim MJ, Park BJ. Reoperation rate after surgery for lumbar herniated intervertebral disc disease: nationwide cohort study. Spine. 2013;38(7):581-590.

10. Johnsson KE, Rosén I, Udén A. The natural course of lumbar spinal stenosis. Clin Orthop Relat Res. 1992;279(279):82-86.

11. Kim KH, Kim YR, Noh SH, et al. Use of acupuncture for pain management in an academic Korean medicine hospital: a retrospective review of electronic medical records. Acupunct Med. 2013;31(2):228-234.

12. Yun K-J, Lee JA, Choi J, et al. A retrospective analysis of patients' conditions using acupuncture in a traditional Korean medicine hospital. Evid Base Comple Alternative Med. 2015;2015(1):1-6.

13. Kim YS, Jun H, Chae Y, et al. The practice of Korean medicine: an overview of clinical trials in acupuncture. Evid Base Comple Alternative Med. 2005;2(3):325-352.

14. Kim K, Jeong Y, Youn Y, et al. Nonoperative Korean medicine combination therapy for lumbar spinal stenosis: a retrospective Case-Series study. Evid Base Comple Alternative Med. 2015;2015(384):1-5.

15. May S, Comer C. Is surgery more effective than non-surgical treatment for spinal stenosis, and which non-surgical treatment is more effective? A systematic review. Physiotherapy. 2013;99(1):12-20.

16. Ammendolia C, Stuber KJ, Rok E, et al. Nonoperative treatment for lumbar spinal stenosis with neurogenic claudication. Cochrane Database Syst Rev. 2013;25(8):CD010712.

17. Brinkhaus Bet al. Acupuncture in patients with chronic low back pain: a randomized controlled trial. Arch Intern Med. 2006;166(4):450-457.

18. Haake M, Müller HH, Schade-Brittinger C, et al. German acupuncture trials (GERAC) for chronic low back pain: randomized, multicenter, blinded, parallel-group trial with 3 groups. Arch Intern Med. 2007;167(17):1892-1898.

19. Thomas KJ, Macpherson H, Ratcliffe J, et al. Longer term clinical and economic benefits of offering acupuncture care to patients with chronic low back pain. Health Technol Assess (Winchester, England). 2005;9(32):1-109.

20. Shin BC, Kim MR, Cho JH, et al. Comparative effectiveness and costeffectiveness of Chuna manual therapy versus conventional usual care for nonacute low back pain: study protocol for a pilot multicenter, pragmatic randomized controlled trial (pCRN study). Trials. 2017;18(1):26.
21. Kim K, Jeong Y, Youn Y, et al. Nonoperative Korean medicine combination therapy for lumbar spinal stenosis: a retrospective Case-Series study. Evid Base Comple Alternative Med. 2015;2015(384):1-5.

22. Mai W, Chen D, Li X. Antioxidant activity of Rhizoma Cibotii in vitro. Adv Pharm Bull. 2012;2(1):107-114.

23. Koh W, Shin JS, Lee J, et al. Anti-inflammatory effect of cortex eucommiae via modulation of the Toll-like receptor 4 pathway in lipopolysaccharide-stimulated RAW 264.7 macrophages. J Ethnopharmacol. 2017;209:255-263.

24. Zhang Z, Dong J, Liu M, et al. Therapeutic Effects of Cortex acanthopanacis Aqueous Extract on Bone Metabolism of Ovariectomized Rats. Evid Base Compl Alternative Med. 2012;2012(5):1-8.

25. Ahn S, Singh P, Jang M, et al. Gold nanoflowers synthesized using Acanthopanacis Cortex extract inhibit inflammatory mediators in LPS-induced RAW264.7 macrophages via NF- $\kappa$ B and AP-1 pathways. Colloids and Surfaces B: Biointerfaces. 2018;162:398-404.

26. Choi HJ, Choi HJ, Park MJ, et al. The inhibitory effects of geranium thunbergii on interferon- $\gamma$ - and LPS-induced inflammatory responses are mediated by Nrf2 activation. Int J Mol Med. 2015;35(5): 1237-1245.

27. Kim K, Shin K-M, Lee J-H, et al. Nonsurgical Korean integrative treatments for symptomatic lumbar spinal stenosis: a Three-Armed randomized controlled pilot trial protocol. Evid Base Compl Alternative Med. 2016;2016:1-7.

28. Park TY, Moon TW, Cho DC, et al. An introduction to Chuna manual medicine in Korea: history, insurance coverage, education, and clinical research in Korean literature. Integr Med Res. 2014;3(2):49-59.

29. Koc Z, Ozcakir S, Sivrioglu K, Gurbet A, Kucukoglu S. Effectiveness of physical therapy and epidural steroid injections in lumbar spinal stenosis. Spine. 2009;34(10):985-989.

30. Jeon C-H, Kim D-J, Kim D-J, Lee H-M, Park H-J. Cross-cultural adaptation of the Korean version of the Oswestry Disability Index (ODI) J Korean Soc Spine Surg. 2005;12(2):146-152.

31. Pratt RK, Fairbank JC, Virr A. The reliability of the shuttle walking test, the Swiss spinal stenosis questionnaire, the Oxford spinal stenosis score, and the Oswestry Disability Index in the assessment of patients with lumbar spinal stenosis. Spine. 2002;27(1):84-91.

32. Kim MH, Cho YS, Uhm WS, Kim S, Bae SC. Cross-cultural adaptation and validation of the Korean version of the EQ-5D in patients with rheumatic diseases. Qual Life Res. 2005;14(5):1401-1406.
Journal of Pain Research

\section{Publish your work in this journal}

The Journal of Pain Research is an international, peer reviewed, open access, online journal that welcomes laboratory and clinical findings in the fields of pain research and the prevention and management of pain. Original research, reviews, symposium reports, hypothesis formation and commentaries are all considered for publication
Dovepress

The manuscript management system is completely online and includes a very quick and fair peer-review system, which is all easy to use. Visit http://www.dovepress.com/testimonials.php to read real quotes from published authors. 